\section{Buchrezension zu: Toxic}

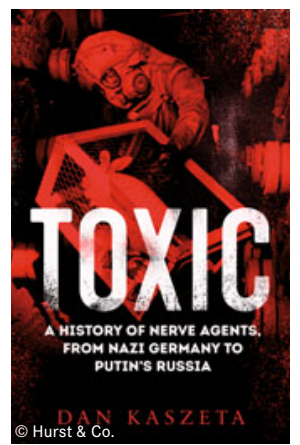

Toxic

A History of Nerve Agents, From Nazi Germany to Putin's Russia

Dan Kaszeta

408 S., 14 Abb., Hurst \& Co., 2020. HC, $25 \mathrm{E}$.

ISBN: 9781787383067

DOI: $10.1007 / \mathrm{s} 12268-020-1475-2$

(C) Der Autor 2020

Irreversible Inhibitoren der Acetylcholinesterase (AChE) führen zu einer Überflutung des menschlichen (und tierischen) Organismus mit dem Neurotransmitter Acetylcholin (ACh), der nicht mehr abgebaut werden kann. Es resultiert ein so genanntes cholinerges Syndrom, das durch eine Parasympathikusaktivierung, Muskelkrämpfe und epileptische Anfälle gekennzeichnet ist. Letztlich kommt es ohne Behandlung mit dem Muskarinrezeptor-Antagonisten Atropin und AChE-Reaktivatoren (Oximen) zu einem qualvollen Tod. Prinzipiell können irreversible AChE-Inhibitoren als Insektizide oder als Gifte für den Menschen verwendet werden.

In dem Buch zeichnet Kaszeta, ein Experte auf dem Gebiet der irreversiblen $\mathrm{AChE}$-Inhibitoren, die historische Entwicklung dieser Wirkstoffgruppe (wegen ihrer Wirkung auf das Nervensystem auch als nerve agents bezeichnet) nach. Die Prototypen dieser Wirkstoffgruppe sind Tabun und Sarin, die mit einem extrem hohen personellen und finanziellen Aufwand während der Naziherrschaft in Deutschland entwickelt wurden. Kaszeta beschreibt sehr eindrücklich, wie diese „Pionierar- beit" des Naziregimes nach dem Zweiten Weltkrieg von den Siegermächten genutzt wurde, selber wirksamere und für die eigenen Soldaten „sicherere“ irreversible AChE-Inhibitoren zu entwickeln. Während die Großmächte irreversible AChE-Inhibitoren nie militärisch eingesetzt haben, wurden sie von weniger mächtigen Ländern wie Irak und Syrien - als "billige“ Waffen eingesetzt. Auch der Einsatz der irreversiblen AChE-Inhibitoren zur gezielten Tötung missliebiger Menschen durch Geheimdienste wird sehr gut dargestellt. Insgesamt ist das Buch von Kaszeta sehr lesenswert. Um es möglichst gut verstehen zu können, sind physiologische und toxikologische Grundkenntnisse unbedingt erforderlich. Diese Grundlagen werden nur recht kursorisch im Appendix A dargestellt. Prinzipiell kann man das Buch problemlos "quer" lesen und sich das herauspicken, was einem am meisten interessiert. Der Rezensent fand die ersten vier Kapitel besonders spannend. Das Buch eignet sich für alle diejenigen, die an (Militär-)Geschichte, Geheimdiensten, Toxikologie sowie fehlgeleiteter menschlicher Intelligenz interessiert sind.

Roland Seifert, Medizinische Hochschule Hannover, Seifert.Roland@mh-hannover.de
* Funding Open Access funding enabled and organized by Projekt DEAL.

* Open Access Dieser Artikel wird unter der Creative Commons Namensnennung 4.0 International Lizenz veröffentlicht, welche die Nutzung, Vervielfältigung, Bearbeitung, Verbreitung und Wiedergabe in jeglichem Medium und Format erlaubt, sofern Sie den/die ursprünglichen Autor(en) und die Quelle ordnungsgemäß nennen, einen Link zur Creative Commons Lizenz beifügen und angeben, ob Änderungen vorgenommen wurden. Die in diesem Artikel enthaltenen Bilder und sonstiges Drittmaterial unterliegen ebenfalls der genannten Creative Commons Lizenz, sofern sich aus der Abbildungslegende nichts anderes ergibt. Sofern das betreffende Material nicht unter der genannten Creative Commons Lizenz steht und die betreffende Handlung nicht nach gesetzlichen Vorschriften erlaubt ist, ist für die oben aufgeführten Weiterverwendungen des Materials die Einwilligung des jeweiligen Rechteinhabers einzuholen. Weitere Details zur Lizenz entnehmen Sie bitte der Lizenzinformation auf http://creativecommons.org/ licenses/by/4.0/deed.de. 